\title{
Kinetic equations for diffusion in the presence of entropic barriers
}

\author{
D. Reguera* and J. M. Rubí \\ Departament de Física Fonamental, Facultat de Física, Universitat de Barcelona, Diagonal 647, 08028 Barcelona, Spain
}

(Received 9 August 2001; published 21 November 2001)

\begin{abstract}
We use the mesoscopic nonequilibrium thermodynamics theory to derive the general kinetic equation of a system in the presence of potential barriers. The result is applied to a description of the evolution of systems whose dynamics is influenced by entropic barriers. We analyze in detail the case of diffusion in a domain of irregular geometry in which the presence of the boundaries induces an entropy barrier when approaching the exact dynamics by a coarsening of the description. The corresponding kinetic equation, named the Fick-Jacobs equation, is obtained, and its validity is generalized through the formulation of a scaling law for the diffusion coefficient which depends on the shape of the boundaries. The method we propose can be useful to analyze the dynamics of systems at the nanoscale where the presence of entropy barriers is a common feature.
\end{abstract}

DOI: 10.1103/PhysRevE.64.061106

PACS number(s): 05.40.Jc, 05.70.Ln, 66.10.Cb

\section{INTRODUCTION}

The free energy landscape of a complex system, when represented as a function of an order parameter or reaction coordinate, presents an intricate aspect consisting of multiple local minima, defining metastable states, separated by barriers. The nature of the barriers depends on which thermodynamic potential varies when passing from one well to the other, and their presence plays an important role in the dynamics of the system. Whereas energy barriers are more frequent in problems of solid state physics, entropy barriers are often encountered in soft condensed matter and biological systems. These barriers may also appear when coarsening the description of a complex system in order to simplify its dynamics. The elimination of some coordinates can be performed by introducing a configurational entropy accounting for the degeneracy (i.e., the number of equivalent configurations) of the state defined by the remaining coordinates. This procedure has been used, for instance, in the context of glasses [1].

The study of the dynamic properties of the system at the mesoscopic level requires a knowledge of the probability distribution function of the relevant degrees of freedom which evolves according to a kinetic equation of the FokkerPlanck type. This equation is usually derived from kinetic theory in the diffusion limit [2-4], from a master equation [5], and from projection operator techniques [6,7].

Our purpose in this paper is to propose a simple theoretical framework to analyze the mesoscopic dynamics of systems in the presence of energetic and entropic barriers. The procedure we follow is based on the application of mesoscopic nonequilibrium thermodynamics [8-18], and was previously used to analyze transport and activated processes in systems governed by a dynamics of the Fokker-Planck type. The kinetic equation follows after obtaining the probability current, occurring in the corresponding continuity equation in the configuration space, from the entropy production accounting from dissipation in that space related to the underlying diffusion process of the probability density. That equa-

\footnotetext{
*Email address: davidr@ precario.ffn.ub.es
}

tion applies irrespectively of the nature of the equilibrium state of the system. When the system is in contact with a heat bath and its volume remains unaltered, in which case the proper thermodynamic potential is the free energy, our equation describes the dynamics in the presence of energetic and entropic barriers. The validity of our method, however, goes beyond this standard situation, and embraces other cases of interest characterized by other thermodynamic potentials and statistical ensembles.

The paper is distributed in the following way. In Sec. II, we present a general derivation of the kinetic equation in the framework of mesoscopic nonequilibrium thermodynamics. This equation governs the evolution of the probability density in the presence of barriers of any nature. In Sec. III we discuss an example in which the presence of an entropic barrier manifests: diffusion in a channel of varying cross section. The corresponding kinetic equation has been referred to as the Fick-Jacobs equation. Section IV is devoted to a derivation of that equation from mesoscopic nonequilibrium thermodynamics, and to the formulation of a scaling law for the diffusion coefficient which is compared to previous results. In Sec. V we extend the results of Sec. IV to the case in which an external field acts on the diffusing particle. Finally, in Sec. VI we present our main conclusions.

\section{KINETIC EQUATIONS IN THE PRESENCE OF POTENTIAL BARRIERS}

Our purpose in this section is to present a general derivation of the kinetic equations describing the evolution in time of the probability density when the statics of the system is characterized by a thermodynamic potential landscape. We consider that the equilibrium state of the system is described by the probability distribution function

$$
P_{e q} \sim \exp \left(\frac{-\Delta \mathcal{W}(\underline{x})}{k T}\right),
$$

where $\mathcal{W}(\underline{x})$ is the minimum reversible work required to change the state of the system [19], which is related to the maximum useful work which can be extracted from it, sometimes referred to as available energy [20] or availability [21]; 
$k$ is Boltzmann's constant, $T$ is the temperature of the medium, and $x$ an arbitrary set of coordinates which may represent the velocity of a particle, the orientation of a spin, the size of a macromolecule or whatever coordinate or order parameter whose values defines the state of the system in a phase space. Variations of the minimum work for a thermodynamic system are expressed as

$$
\Delta \mathcal{W}=\Delta U-T \Delta S+p \Delta V-\mu \Delta N+y \Delta Y+\cdots,
$$

where $U$ is the internal energy, $S$ is the entropy, $V$ is the volume, and $N$ is the number of particles of the system, whereas $T, p$, and $\mu$ are the temperature, pressure and chemical potential of the environment. The term $y \Delta Y$ represent other kinds of work (electric, magnetic, surface work, etc.) performed on the system, $y$ being the intensive parameter and $Y$ its conjugate extensive variable [22]. For the case of constant temperature, volume, and number of particles, the minimum work corresponds to the Helmholtz free energy $F$. In general, that quantity reduces to the thermodynamics potentials by imposing corresponding constraints on Eq. (2) [22].

Having specified the statics of the system, we will derive the kinetic equation describing the evolution of the probability density. To this end, we will use the framework of mesoscopic nonequilibrium thermodynamics. This theory applies the scheme of nonequilibrium thermodynamics to describe the dynamics of mesoscopic degrees of freedom. The treatment of a diffusion process in the framework of nonequilibrium thermodynamics is extended to the case in which the relevant quantity is a probability density, defined in phase space, instead of a mass density. The starting point is then the formulation of the Gibbs equation

$$
\delta S=-\frac{1}{T} \int \mu(\underline{x}) \delta P(\underline{x}, t) d \underline{x},
$$

which resembles the corresponding law proposed in nonequilibrium thermodynamics in terms of the mass density of particles. Here $\mu(\underline{x})$ is a generalized chemical potential, whereas $P(\underline{x}, t)$ is the probability density.

This expression is compatible with the Gibbs entropy

$$
S(t)=-k \int P\left(\underline{x, t)} \ln \frac{P(\underline{x}, t)}{P_{e q}(\underline{x})} d \underline{x}+S_{e q},\right.
$$

where $S_{e q}$ is the entropy when the system and the heat bath are at equilibrium. Effectively, taking variations in Eq. (4), and using the expression of the chemical potential per particle [9],

$$
\mu(\underline{x})=k T \ln \frac{P(\underline{x}, t)}{P_{e q}(\underline{x})}+\mu_{e q},
$$

where $\mu_{e q}$ is the chemical potential at equilibrium, one arrives at expression (3).

From the Gibbs equation we can obtain the entropy production related to the underlying diffusion process in $\underline{x}$ space. Calculating the time derivative of the entropy from Eq. (4), one has

$$
\frac{\partial S}{\partial t}=-k \int \ln \frac{P}{P_{e q}} \frac{\partial P}{\partial t} d x .
$$

Quite generally, we may assume that the probability density satisfies the continuity equation

$$
\frac{\partial P}{\partial t}=-\frac{\partial}{\partial \underline{x}} J
$$

where $J(x, t)$ is a current defined in $\underline{x}$ space which has to be determined. Now substituting the time derivative of Eq. (7) into Eq. (6), one obtains the entropy production

$$
\sigma=-k \int \underline{J}(\underline{x}, t) \frac{\partial}{\partial \underline{x}} \ln \frac{P}{P_{e q}} d x .
$$

To arrive at Eq. (8) we have also performed a partial integration, and assumed that the current vanishes at the boundaries of the system in $x$ space.

In the nonequilibrium thermodynamics scheme, the entropy production [Eq. (8)] consists of contributions of products between the current $J(\underline{x}, t)$ and its conjugated thermodynamic force $-k(\partial / \partial x) \overline{\ln }\left(P / P_{e q}\right)$, for each value of the coordinate $x$. Assuming locality in $x$ space, for which only currents and forces at the same value of $x$ are coupled, one obtains the linear law

$$
\underline{J}=-k L(\underline{x}) \frac{\partial}{\partial \underline{x}} \ln \frac{P}{P_{e q}},
$$

in which the phenomenological coefficient $L(\underline{x})$ may in general depend on $x$.

The resulting kinetic equation then follows by substituting Eq. (9) into the continuity equation (7),

$$
\frac{\partial P}{\partial t}=\frac{\partial}{\partial \underline{x}}\left(D P \frac{\partial}{\partial \underline{x}} \ln \frac{P}{P_{e q}}\right),
$$

where we have defined the diffusion coefficient

$$
D(\underline{x})=\frac{k L(\underline{x})}{P} .
$$

This equation, which in view of Eq. (1) can also be written as

$$
\frac{\partial P}{\partial t}=\frac{\partial}{\partial \underline{x}}\left(D \frac{\partial P}{\partial \underline{x}}+\frac{D}{k T} \frac{\partial \Delta \mathcal{W}}{\partial \underline{x}} P\right),
$$

is the Fokker-Planck equation accounting for the evolution of the probability density in $x$ space.

Under the conditions for which $\mathcal{W}=F=U-T S$, this equation transforms into the Fokker-Planck equation for a system in the presence of energy and entropy barriers. One then obtains

$$
\frac{\partial P}{\partial t}=\frac{\partial}{\partial \underline{x}}\left(D \frac{\partial P}{\partial \underline{x}}+\frac{D}{k T} \frac{\partial \Delta U}{\partial \underline{x}} P-\frac{D}{k} \frac{\partial \Delta S}{\partial \underline{x}} P\right),
$$


where the drift term consists of contributions due to an external potential and to variations of the entropy. When the barrier is purely energetic, only the first contribution remains, and the previous equation reduces to the well-known Fokker-Planck equation

$$
\frac{\partial P}{\partial t}=\frac{\partial}{\partial \underline{x}}\left(D \frac{\partial P}{\partial \underline{x}}+\frac{D}{k T} \frac{\partial \Delta U}{\partial \underline{x}} P\right) .
$$

If the nature of the barrier is purely entropic, Eq. (13) then corresponds to the Fick-Jacobs equation

$$
\frac{\partial P}{\partial t}=\frac{\partial}{\partial \underline{x}}\left(D \frac{\partial P}{\partial \underline{x}}-\frac{D}{k} \frac{\partial \Delta S}{\partial \underline{x}} P\right),
$$

first derived in the context of diffusion of Brownian particles in a channel of nonconstant cross section [23].

The general form of Eq. (12), in which the equilibrium distribution function does not need to be specified and is given in general by Eq. (1), makes that result applicable to a great diversity of situations. For example, for a Brownian particle, for which the minimum work is simply its kinetic energy, the equation corresponds to the usual Fokker-Planck equation. When $x$ is an order parameter or a reaction coordinate, Eq. (12) provides the kinetic equation in the presence of barriers. The method used in this section then offers a common formalism able to analyze the dynamics of a system in the presence of energy and entropy barriers. In this paper, we will mainly focus our analysis to the case in which the potential is strictly entropic.

\section{DIFFUSION THROUGH A CHANNEL OF VARYING CROSS SECTION: FICK-JACOBS EQUATION}

The influence that the presence of an entropic barrier exerts on the dynamics of a system can be illustrated by deriving the kinetic equation governing Brownian diffusion through a channel of varying cross-section. This equation was first proposed by Jacobs [23] and subsequently rederived by Zwanzig [24] on the basis of more fundamental arguments.

\section{A. Jacobs' derivation}

Jacobs in his book Diffusion Processes [23], provided an heuristic derivation of the equation governing diffusion in a symmetric tube whose cross section $A(x)$ varies along the $x$ axis, defined by the center line of the tube. The argument runs as follows.

Consider an elementary volume of thickness $d x$ perpendicular to the axis of the tube. The total amount of particles in this slice, at $x$ and time $t$ is $C(x, t) d x$, which is the integral of the concentration over the volume $A(x) d x$. The rates of entrance and exit of the diffusing substance into this volume are given by the Fick's law $J=-D_{0}((\partial C / A) / \partial x)$ where $D_{0}$ is the diffusion coefficient, and $C / A$ is the local volume concentration. In this case, both rates are different not only because the concentration gradient $\partial C / \partial x$ changes with the distance, but also due to the variation of the cross-section of the channel. Explicitly, the rate of entrance into this elementary volume is $-D_{0} A((\partial C / A) / \partial x)$, whereas the rate of exit is given by

$$
-D_{0}\left[A \frac{\partial C / A}{\partial x}+\frac{\partial}{\partial x}\left(A \frac{\partial C / A}{\partial x}\right) d x+\cdots\right] .
$$

The difference between both rates provides the rate of change of the substance in the elementary volume, which can also be expressed as $(\partial C / \partial t) d x$. Neglecting quadratic terms in $d x$, one easily arrives at the equation governing the diffusion in the channel,

$$
\frac{\partial C}{\partial t}=D_{0} \frac{\partial}{\partial x}\left[A \frac{\partial}{\partial x}\left(\frac{C}{A}\right)\right]=D_{0} \frac{\partial}{\partial x}\left(\frac{\partial C}{\partial x}-\frac{1}{A(x)} \frac{d A(x)}{d x} C\right),
$$

which is referred to as the Fick-Jacobs equation.

\section{B. Zwanzig's derivation}

Zwanzig in Ref. [24] reported a more general and rigorous derivation of the Fick-Jacobs equation. For the sake of simplicity, let us review the two-dimensional (2D) case. The method consists of performing a reduction in the number of coordinates from the 2D Smoluchowski equation to a 1D description.

The starting point is the two-dimensional Smoluchowski equation for diffusion through a general potential $U(x, y)$,

$$
\begin{gathered}
\frac{\partial c(x, y, t)}{\partial t}=D_{0} \frac{\partial}{\partial x} e^{-\beta U(x, y)} \frac{\partial}{\partial x} e^{\beta U(x, y)} c(x, y, t) \\
+D_{0} \frac{\partial}{\partial y} e^{-\beta U(x, y)} \frac{\partial}{\partial y} e^{\beta U(x, y)} c(x, y, t),
\end{gathered}
$$

where $\beta=1 / k T$, and $c(x, y, t)$ is the concentration. To perform the reduction to one-dimension, this equation is integrated over the variable $y$, leading to

$$
\frac{\partial C(x, t)}{\partial t}=D_{0} \frac{\partial}{\partial x} \int e^{-\beta U(x, y)} \frac{\partial}{\partial x} e^{\beta U(x, y)} c(x, y, t) d y,
$$

where the reduced concentration is defined as

$$
C(x, t)=\int c(x, y, t) d y \text {. }
$$

The key point of the derivation is the assumption of equilibration in the transverse direction. Under this assumption, one can define an averaged $x$-dependent free energy $F(x)$ through the expression

$$
e^{-\beta F(x)}=\int e^{-\beta U(x, y)} d y,
$$

from which one can define a normalized conditional probability distribution 


$$
\rho(x ; y)=\frac{e^{-\beta U(x, y)}}{e^{-\beta F(x)}} .
$$

Then, under the local equilibrium approximation, the concentration $c(x, y, t)$ factorizes as follows:

$$
c(x, y, t) \cong C(x, t) \rho(x ; y) .
$$

Taking these considerations into account, one finally obtains

$$
\frac{\partial C(x, t)}{\partial t} \cong \frac{\partial}{\partial x} D_{0} e^{-\beta F(x)} \frac{\partial}{\partial x} e^{\beta F(x)} C(x, t),
$$

which constitutes the generalization of the Fick-Jacobs equation (16) to the case of a two dimensional potential $U(x, y)$.

In the previous analysis, we have not taken into account the fact that the normal flux must vanish at the boundaries. The role played by this zero normal flux conditions can be replaced by the confining potential $U(x, y)$. In fact, if $U(x, y)$ is a boxlike potential, i.e., zero inside the tube and infinite outside, it is clear that $e^{-\beta F(x)}=2 w(x)$, being $w(x)$ the half width of the tube. In this case, the barrier is purely entropic, and one recovers the usual Fick-Jacobs equation. The extension to the three-dimensional case is quite straightforward by taking into account that in this case the integration in the transverse coordinates involves variables $y$ and $z$, and the width of the 2D tube must be replaced by the transverse section $\pi w(x)^{2}$.

Zwanzig's analysis clearly manifests that the accuracy of the Fick-Jacobs equation is conditioned on the existence of local equilibrium in the transverse direction. The author analyzed the effect of the deviations from the local equilibrium related to the variations of the density $\delta c(x, y, t) \equiv c(x, y, t)$ $-C(x, t) \rho(x ; y)$. He derived an equation for the evolution of these deviations, which suggested that the accuracy of the Fick-Jacobs equations is restricted to situations verifying $\left|w^{\prime}(x)\right|<1$, that is, when the section of the tube varies smoothly. In addition, he showed that the range of validity of the one-dimensional Fick-Jacobs description could be extended by introducing a position-dependent effective diffusion coefficient

$$
D_{Z}(x)=D_{0} \frac{1}{1+\gamma w^{\prime}(x)^{2}},
$$

where $D_{0}$ is the molecular diffusion coefficient, and the parameter $\gamma$ depends on the dimensionality. The explicit expression for this coefficient is obtained through an expansion in powers of $w^{\prime}(x)$ [24]. The result is

$$
D(x)=D_{0}\left[1-\gamma w^{\prime}(x)^{2}+\cdots\right],
$$

where

$$
\gamma=\frac{\int \nu^{2} e^{-\beta V(\nu)} d \nu}{\int e^{-\beta V(\nu)} d \nu}
$$

$\nu$ being the transverse coordinate scaled by the function $w(x)$ [i.e., $\nu=y / w(x)$ in two-dimensions, and $\nu=r / w(z)$ for a 3D tube of radius $r$ with cylindrical symmetry], and $V(\nu)$ $=U(x, y)$. For a purely confining potential, the results obtained by Zwanzig are $\gamma=1 / 3$ for the $2 \mathrm{D}$ case, and $\gamma=1 / 2$ for a 3D tube with cylindrical symmetry. From expansion (26) he infers expression (25), based merely on the fact that this reconstruction of the series improves the agreement with the exact results.

\section{FICK-JACOBS EQUATION FROM MESOSCOPIC NONEQUILIBRIUM THERMODYNAMICS}

For the case of an enclosure of varying cross section, the concept of entropic barrier is remarkably simple. At equilibrium, the density of diffusing material $\rho_{0}$ is constant. If we contract the $3 \mathrm{D}$ description retaining the coordinate $x$, the resulting $1 \mathrm{D}$ equilibrium distribution is

$$
\rho_{e q}(x)=\int \rho_{0} d y d z=\rho_{0} A(x) .
$$

In this case, the diffusing particle constitute an isolated system. Therefore, its corresponding thermodynamic potential obtained from Eq. (2) is simply $\Delta \mathcal{W}=-T \Delta S$, where the entropic barrier is, in accordance with Eq. (1),

$$
\Delta S(x)=k \ln A(x) .
$$

The previous equation clearly manifests that the entropic barrier originates from the variation of the space available for the diffusing particles. Note that Eq. (29) corresponds to the usual microcanonical definition of the entropy in terms of the number of states which in this case is simply proportional to the area of the tube.

Once identified the equilibrium distribution (or the entropic barrier), the kinetic equation describing diffusion in the presence of the entropic barrier follows from our general scheme developed in Sec. II. According to Eq. (15), the kinetic equation is

$$
\frac{\partial P}{\partial t}=\frac{\partial}{\partial x}\left(D(x) \frac{\partial P}{\partial x}-\frac{D(x)}{A(x)} \frac{\partial A(x)}{\partial x} P\right),
$$

which has the same structure as the Fick-Jacobs equation, but with a spatially dependent diffusion coefficient.

At this point, it is worth analyzing the role played by this coefficient. In Ref. [24] it was shown that the validity of the 1D description could be extended if we use an effective spatially dependent diffusion coefficient. However, the agreement one can achieve with expression (25) proposed by Zwanzig is not always satisfactory. In particular, for the $3 \mathrm{D}$ example discussed in Ref. [24], results for the current of particles obtained by using the expression of the diffusion coefficient [Eq. (25)] present severe discrepancies with the corresponding expression coming from the exact result of the 3D Smoluchowski equation, when the section of the tube changes abruptly.

The appearance of an entropic barrier originates from the reduction of the space to a single coordinate. But this reduc- 
tion may also have implications on the form of the diffusion coefficient. The molecular diffusion coefficient in the real space, $D_{0}$, gives information about the dispersion of the displacement. In two-dimensions, we can estimate

$$
D_{0} \sim \frac{(\Delta \mathbf{r})^{2}}{t}=\frac{\Delta x^{2}\left[1+\left(\frac{\Delta y}{\Delta x}\right)^{2}\right]}{t},
$$

which manifests that the diffusion coefficient involves the displacement in both coordinates. Once we have contracted the $y$ coordinate, the resulting effective diffusion coefficient only depends on the dispersion in the remaining $x$ coordinate, that is qualitatively $D_{e f f} \sim(\Delta x)^{2} / t$. Therefore, from Eq. (31) we can infer the behavior $D_{\text {eff }} \sim D_{0} /\left[1+(\Delta y / \Delta x)^{2}\right]$. The previous equation provides a hint about the fact that the reduction of coordinates may involve not only the appearance of an entropic barrier, but also a scaling of the diffusion coefficient. Following this heuristic reasoning, we will propose a scaling law

$$
D_{s}(x)=D_{0} \frac{1}{\left(1+y^{\prime}(x)^{2}\right)^{\alpha}}
$$

for the diffusion coefficient appearing in Eq. (30), where $y^{\prime}(x)=d y / d x$, and $y(x)=w(x)$ defines the shape of the enclosure. The objective is then to test if the use of this expression is able to extend the range of validity of the 1D FickJacob-type description.

The values of the scaling exponents can be estimated from the calculations performed in Ref. [24]. We can expand our expression for the diffusion coefficient $D_{s}(x)$ in terms of $w^{\prime}(x)^{2}$, and compare the result with the one obtained by Zwanzig [Eq. 26]. From this comparison, one obtains that a reasonable choice of the scaling exponents is $\alpha=1 / 3$ for the $2 \mathrm{D}$ case and $\alpha=1 / 2$ for the case of a $3 \mathrm{D}$ tube.

In the remaining of this section, we will test this result for the two cases discussed in Ref. [24] which admit an exact solution. We will see that the agreement with the exact result, when we use our effective diffusion coefficient given by Eq. (32), is significantly improved.

\section{A. Effective diffusion coefficient in a $2 \mathrm{D}$ periodic symmetric channel}

The first case under scrutiny is the diffusion in a $2 \mathrm{D}$ periodic channel defined by

$$
x=u+a \cosh v \sin u, \quad y=v+a \sinh v \cos u,
$$

where diffusion occurs in the region comprised between 0 $<u<2 \pi$ and $-V<v<V$. The parameter $\lambda \equiv a \cosh V$ must be smaller than 1 , to avoid double valuation of the walls. Figure 1 illustrates the shape of the tube for $V=0.5$ and $a=0.5$.

At very long times, diffusion in periodic channels can be described through an effective diffusion coefficient $D^{*}$, ac-

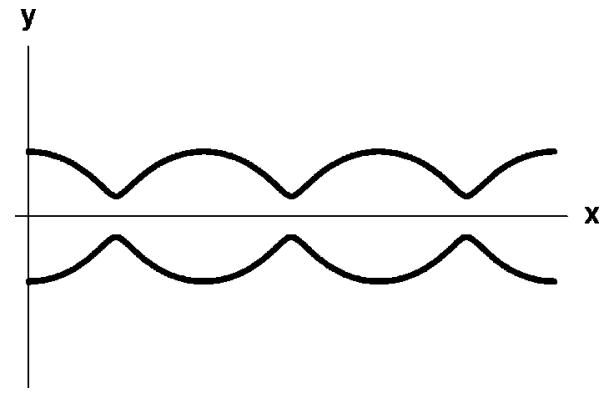

FIG. 1. Shape of the two-dimensional periodic channel defined parametrically by Eq. (33), for $V=0.5$ and $a=0.5$.

counting for the effects of passing through many constrictions in the channel. This effective coefficient can be evaluated as [25]

$$
\frac{1}{D^{*}}=\left\langle\frac{1}{D(x) w(x)}\right\rangle\langle w(x)\rangle
$$

where \langle\rangle denotes average over one period of the potential.

For this particular geometry, an exact expression for $D^{*}$ was derived in Ref. [25]

$$
\left(\frac{D^{*}}{D_{0}}\right)_{\text {exact }}=\left[1+\frac{\lambda^{2}}{2} \frac{\tanh V}{V}\right]^{-1},
$$

and was compared to the results obtained with the FickJacobs equation using a constant diffusion coefficient $D_{0}$, and with the position-dependent diffusion coefficient $D_{Z}(x)$.

In Fig. 2 we represent the results for $D^{*}$ obtained using the different approaches mentioned above, plus the ones corresponding to our expression $D_{s}(x)$. One can easily realize that the results obtained with the expression of $D_{s}(x)$ we propose improve the ones when using $D_{Z}(x)$ and constitute an excellent approximation of the exact result.

\section{B. Steady-state flux through a 3D hyperboloidal cone}

The improvement of the results one can achieve with $D_{s}(x)$ is more remarkable for the $3 \mathrm{D}$ example discussed in

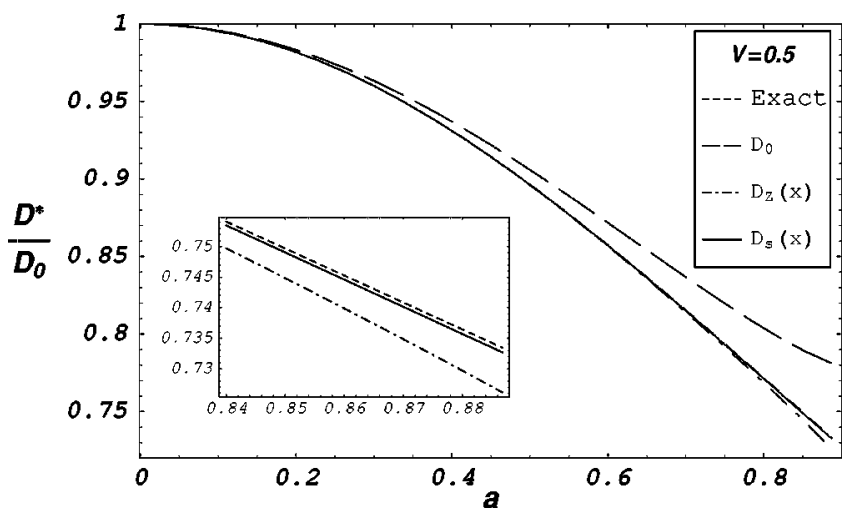

FIG. 2. Effective diffusion coefficient $D^{*}$ for the 2D channel for $V=0.5$ as a function of the parameter $a$, using different expressions for the diffusion coefficient. The inset is a zoom to illustrate the accuracy of the different approaches. 


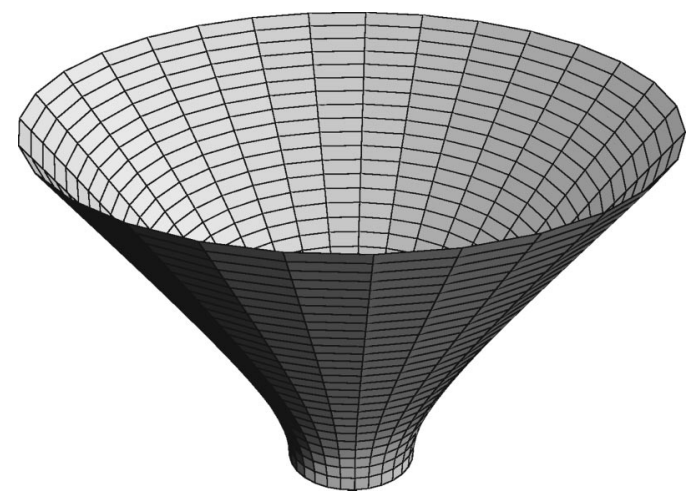

FIG. 3. 3D hyperboloidal cone for $a=1,0<\xi<5$ and $\eta_{0}$ $=0.5$.

Ref. [24]. In this case, the shape of the tube corresponds to a 3D hyperboloidal cone as the one depicted in Fig. 3. It can be conveniently described by using oblate spheroidal coordinates $(\xi, \eta, \varphi)$, related to the cylindrical coordinates $(r, z, \varphi)$ by

$$
r^{2}=a^{2}\left(\xi^{2}+1\right)\left(1-\eta^{2}\right), \quad z=a \xi \eta
$$

In this coordinate system, $\xi=0$ corresponds to the small hole and $\xi=\infty$ to the far end of the tube; $\eta=1$ is the $z$ axis, $\eta$ $=0$ is the $(x, y)$ plane, whereas $\eta=\eta_{0}$ corresponds to the surface of the tube. Diffusion then takes place in the region

$$
0<\xi<\infty, \quad \eta_{0}<\eta<1, \quad 0<\varphi<2 \pi
$$

The steady-state 3D diffusion equation can be solved by using the boundary conditions $C=0$ for $\xi=0, C=C_{0}$ for $\xi$ $=\infty$, and the condition that the normal flux vanishes at the walls $\eta=\eta_{0}$. In this situation, the exact steady-state flux through the exit hole $\xi=0$ is [24]

$$
J_{\text {exact }}=4 D a\left(1-\eta_{0}\right) C_{0} .
$$

Alternatively, one can use the Fick-Jacobs equation derived in Sec. III to calculate that flux, yielding

$$
J=C_{0}\left[\int_{0}^{\infty} d z \frac{1}{D(z) A(z)}\right]^{-1} .
$$

The results are depicted in Fig. 4, corresponding to the "exact," unmodified Fick-Jacobs equation, in which $D(z)=D_{0}$ is a constant, and to the choices $D_{Z}(z)$ and $D_{s}(z)$. From the examples we have discussed, it becomes clear that our proposal of a scaling law for the spatially dependent diffusion coefficient leads to a better agreement with the exact results.

\section{DIFFUSION IN A CHANNEL OF VARYING CROSS SECTION UNDER THE INFLUENCE OF A FIELD}

In Sec. II, we have discussed the effect of entropic barriers in the dynamics. The presence of these barriers is sometimes accompanied by energy barriers. The theory we have developed admits a generalization to the case in which both entropic and energetic barriers coexist. This will be the purpose of this section.

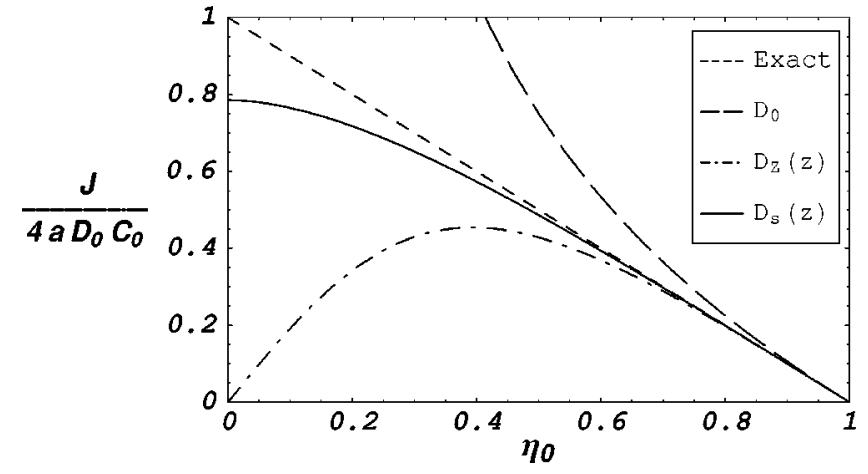

FIG. 4. Comparison of steady state fluxes through an arbitrary hyperboloidal cone, against the value of $\eta_{0}$.

An illustrative example of that situation is the problem of diffusion in a 2D channel of nonconstant cross-section in the presence of gravity. The situation is depicted schematically in Fig. 5.

If gravity is involved, the $2 \mathrm{D}$ equilibrium distribution is given by the usual Boltzmann factor

$$
P_{e q}(x, y) \sim e^{-m g y / k T} .
$$

Considering the normalization condition

$$
1=\int P_{e q}(x, y) d x d y=\int P_{e q}(x) d x,
$$

and using expression (40), one can identify the reduced 1D equilibrium distribution as

$$
\begin{aligned}
P_{e q}(x) & \sim \int_{y_{1}(x)}^{y_{2}(x)} d y \exp \left(\frac{-m g y}{k T}\right) \\
& =\frac{k T}{m g} \exp \left(\frac{-m g y_{1}(x)}{k T}\right)\left[1-\exp \left(\frac{-2 m g w(x)}{k T}\right)\right],
\end{aligned}
$$

where $y_{1}(x)$ and $y_{2}(x)$ define the boundaries of the channel and $2 w(x)=y_{2}(x)-y_{1}(x)$ is its width. The dynamic equation then follows after introducing $P_{e q}(x)$ in Eq. (10), yielding

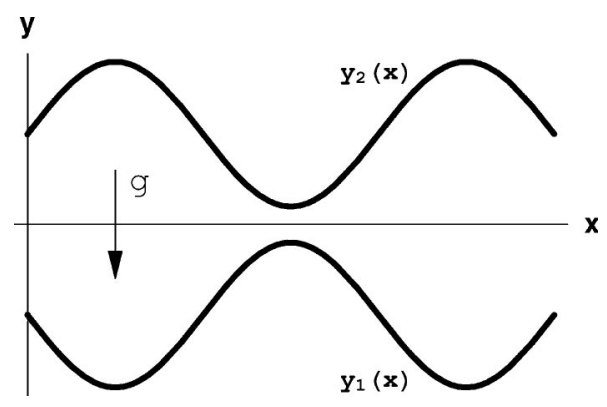

FIG. 5. Schematic representation of the $2 \mathrm{D}$ tube in the presence of gravity. 


$$
\frac{\partial P}{\partial t}=\frac{\partial}{\partial x}\left[D(x) \frac{\partial P}{\partial x}+\frac{D(x) m g}{k T} y_{1}^{\prime}(x) \operatorname{coth}\left(\frac{m g w(x)}{k T}\right) P\right]
$$

for the case of a symmetric channel for which $y_{2}(x)=$ $-y_{1}(x)$. From the previous expressions one can distinguish different limiting cases.

(1) In the limit $\epsilon \equiv m g w(x) / k T \ll 1$, we can expand the reduced equilibrium distribution [Eq. (42) ] in terms of $\epsilon$,

$$
\begin{aligned}
P_{e q}(x) & \sim \frac{k T}{m g}[1+O(\epsilon)]\left(1-1+\frac{2 m g w(x)}{k T}+O\left(\epsilon^{2}\right)\right) \\
& \sim 2 w(x),
\end{aligned}
$$

recovering the case of purely entropic barrier discussed in Sec. IV. Equation (43) reduces then to the Fick-Jacobs equation $(30)$.

(2) For the case $\epsilon \gg 1$, gravity dominates, coth $\epsilon \rightarrow 1$, and we recover the dynamics for diffusion along a 1D purely energetic barrier:

$$
\frac{\partial P}{\partial t}=\frac{\partial}{\partial x}\left(D(x) \frac{\partial P}{\partial x}+\frac{D(x) m g}{k T} y_{1}^{\prime}(x) P\right) .
$$

(3) If the boundaries are flat, that is, $y_{1}(x)=-y_{2}(x)$ $=c t e$, the equilibrium distribution $P_{e q}(x)$ is then constant, and the dynamics can be properly described by the 1D Smoluchowski equation:

$$
\frac{\partial P}{\partial t}=\frac{\partial}{\partial x}\left(D(x) \frac{\partial P}{\partial x}\right)
$$

The evolution of this confined system in the presence of gravity dictated by Eq. (43) presents some peculiarities. On the one hand, since the energy barrier depends on the coordinate we have eliminated, there exists a coupling between entropy and energy barriers and not a mere superposition of the drifts related to each of them, which is reflected by the presence of the term $\operatorname{coth}[m g w(x) / k T]$ in the drift of Eq. (43). On the other hand, it is important to highlight that Eq. (43) does not satisfy the detailed balance condition: the mobility $b \equiv[D(x) / k T] \operatorname{coth}[m g w(x) / k T]$ and the diffusion $D(x)$ are not related through the usual Einstein relation $D(x)=k T b$. That is, the signature that the fluctuation-dissipation theorem, which holds at equilibrium, may lose its validity when we perform a reduction of the variables describing the state of the system.

Concerning the effective diffusion coefficient, a scaling law like the one proposed through Eq. (32), which is valid in absence of external forces, will in general not be correct when an external field is present. The value of the scaling exponent $\alpha$ varies in the presence of external potentials, since the scaling exponents changes due to interactions. The range of validity of the Fick-Jacobs description, and the modifications in the scaling of the effective diffusion coefficient under the presence of external fields, requires a more elaborated treatment.

\section{CONCLUSIONS}

In this paper we have presented a theory to describe the kinetics of a system whose equilibrium state is characterized by a given landscape of an unspecified thermodynamic potential. The theory is based on mesoscopic nonequilibrium thermodynamics, which uses the scheme of nonequilibrium thermodynamics [9] at the mesoscopic level of description in which the pertinent fields are probability densities.

In particular, we have established a kinetic equation for a system in the presence of entropy barriers. The barriers may be inherent to the intimate structure of the system or may emerge as a consequence of the elimination procedure of some coordinates when one tries to simplify its dynamic description.

An illustrative example treated in the literature is the diffusion of a particle in a region of irregular geometry. The governing equation for the probability density is known as the Fick-Jacobs equation, and was derived heuristically by Jacob [23] and directly from the proper coordinate reduction procedure by Zwanzig [24]. In our derivation, the entropic barrier directly comes out from the Boltzmann entropy, which follows from the proper accounting of the number of accessible states of the system. In the kinetic equation we have obtained: the dependency of the diffusion coefficient on the coordinate follows from the general dependency of the Onsager transport coefficients on the state variable, according to the rules of nonequilibrium thermodynamics. This dependency becomes crucial in the reduced description accurately resembling the exact solution. We have proposed a scaling law for the diffusion coefficient, reaching a very good agreement with the exact solution even in the case when the original Fick-Jacobs equation does not provide a good approximation. In this way, we have placed the validity of a Fick-Jacob-like description in a more general context.

The theory we have proposed can also be applied to cases in which an energy barrier is also present. We have obtained the corresponding Fokker-Planck equation describing its dynamics. Interesting characteristics of this equation are the coupling of the entropy and energy barriers and the apparent violation of the fluctuation dissipation theorem, resulting from the elimination of variables.

The theory we have presented is applicable to a wide variety of systems of different nature. Apart from the diffusion problem in the presence of an entropic barrier we have discussed in this paper, we can quote protein folding [26], glassy systems [27], transport of ions [28] and macromolecules [29-32] through membranes or channels, motion of polymers subjected to rigid constraints [33], protein binding kinetics [34,35], drug release [36], nucleation [37], or polymer crystallization [38], to mention just a few examples in which the presence of entropic barriers becomes relevant.

There still remains open questions whose answers go beyond the scope of this paper. The main pitfalls arise from the coordinate reduction procedure. The question is how to proceed with the coarsening in more complex situations. For instance, it will be desirable to analyze the effect of asymmetries either intrinsic to the nature of the landscape or due 
to the presence of external forces which impose a preferred direction, which occurs in many situations of practical interest. Another interesting point will be to analyze the behavior when the slope diverges, giving rise to regions in which the particle may become trapped, thus breaking the ergodicity of the system. The solutions to these problems probably demand a more elaborate analysis of the scaling of the diffusion coefficient which takes these effects into account. Just proceeding systematically along the lines we have established by means of our theory, one could arrive at a more complete theoretical framework providing a dynamic description of systems exhibiting entropic barriers.

\section{ACKNOWLEDGMENTS}

We want to acknowledge P. Mazur, J. M. Vilar, P. Reimann, and G. Schmidg for their comments and suggestions. D.R. wishes to thank Generalitat de Catalunya for financial support. This work was partially supported by DGICYT of the Spanish Government under Grant No. PB98-1258.
[1] F.H. Stillinger and T.A. Weber, Phys. Rev. A 25, 978 (1982); Science 225, 983 (1984).

[2] J. A. McLennan, Introduction to Non-equilibrium Statistical Mechanics (Prentice Hall, Englewood Cliffs, NJ, 1989).

[3] E. M. Lifshitz and L. P. Pitaevskii, Physical Kinetics (Pergamon Press, Oxford, 1981).

[4] D. N. Zubarev, Non-equilibrium Statistical Thermodynamics (Consultants Bureau, New York, 1974).

[5] N. G. van Kampen, Stochastic Processes in Physics and Chemistry (North-Holland, Amsterdam, 1992).

[6] N.G. van Kampen and I. Oppenheim, Physica A 138, 231 (1986).

[7] J.M. Deutch and I. Oppenheim, Faraday Discuss. Chem. Soc. 83, 1 (1987).

[8] I. Prigogine and P. Mazur, Physica (Amsterdam) XIX, 241 (1953).

[9] S. R. de Groot and P. Mazur, Non-equilibrium Thermodynamics (Dover, New York, 1984).

[10] A. Pérez-Madrid, J.M. Rubí, and P. Mazur, Physica A 212, 231 (1994).

[11] I. Pagonabarraga, A. Pérez-Madrid, and J.M. Rubí, Physica A 237, 205 (1997).

[12] J.M. Rubí and P. Mazur, Physica A 250, 253 (1998).

[13] J.M. Rubí and A. Pérez-Madrid, Physica A 264, 492 (1999).

[14] P. Mazur, Physica A 261, 451 (1998).

[15] P. Mazur, Physica A 274, 491 (1999).

[16] J.M. Rubí and P. Mazur, Physica A 276, 477 (2000).

[17] D. Reguera, J.M. Rub, and A. Pérez-Madrid, Physica A 259, 10 (1998).
[18] I. Santamara-Holek, D. Reguera, and J.M. Rub, Phys. Rev. E 63, 051106 (2001).

[19] L. D. Landau and E. M. Lifshitz, Course of Theoretical Physics Vol 5 (Statistical Physics Part 1) (Pergamon Press, New York, 1980).

[20] J. W. Gibbs, Scientific Papers Vol 1: Thermodynamics (Longman, New York, 1906), pp. 49-54.

[21] J. H. Keenan, Thermodynamics (Wiley, New York, 1941) p. 290.

[22] H. Reiss. Methods of Thermodynamics (Dover, New York, 1965).

[23] M. H. Jacobs. Diffusion Processes (Springer, New York, 1967).

[24] R. Zwanzig, J. Phys. Chem. 96, 3926 (1992).

[25] R. Zwanzig, Physica A 117, 277 (1983).

[26] C.J. Camacho, Phys. Rev. Lett. 77, 2324 (1996).

[27] U. Mohanty, I. Oppenheim, and C.H. Taubes, Science 266, 425 (1994).

[28] P. Gates, K. Cooper, J. Rae, and R. Eisenberg, Prog. Biophys. Mol. Biol. 53, 153 (1990).

[29] J. Han and H.G. Craighead, Science 288, 1026 (2000).

[30] L. Liu, P. Li, and S.A. Asher, Nature (London) 397, 141 (1999).

[31] W. Sung and P.J. Park, Phys. Rev. Lett. 77, 783 (1996).

[32] G.W. Slater, H.L. Guo, and G.I. Nixon, Phys. Rev. Lett. 78, 1170 (1997).

[33] M. Fixman, J. Chem. Phys. 69, 1527 (1978).

[34] N. Agmo and G.M. Sastry, Chem. Phys. 212, 207 (1996).

[35] S.-Y. Sheu and D.-Y. Yang, J. Chem. Phys. 112, 408 (2000).

[36] R.A. Siegel, J. Controlled Release 69, 109 (2000).

[37] F.G. Shi and K.N. Tu, Phys. Rev. Lett. 74, 4476 (1995).

[38] J.P.K. Doye and D. Frenkel, J. Chem. Phys. 110, 7073 (1999). 